\title{
Hyphomycetes in rain water, melting snow and ice
}

\author{
BAZYLI CZECZUGA and MIROSŁAWA ORŁOWSKA \\ Department of General Biology, Academy of Medicine \\ Kilinskiego 1, PL-15-230 Bialystok, Poland
}

C zeczuga B., O r low s k a M.: Hyphamyceles in rain water, melting snow and ice. Acta Mycol. 34 (2): $181-200,1999$.

The investigations performed by direct microscopy method or bait technique (fragments of plants, cellophane, snake skin) using rain water flowing from trees, different types of roofs and melting snow and ice allowed determination of 146 species, including 55 new to Poland. Acro-aquatic species were predominant and only several belonged to typically aquatic Hyphomycetes.

Key words: Hyphomycetes, freshwater fungi, aero-aquatic fungi.

\section{INTRODUCTION}

Fungi of the Hyphomycetes demonstrate a great adaptive ability and are thus widely spread in nature. They show a great tolerance to temperature and marked resistance to varied conditions of wet and dry periods.

Analysis of the freshwater Hyphomycetes with respect to ecology shows their considerable heterogeneity. Apart from the species, which grow in the aquatic environment the group also includes areo-aquatic and terrestrial-aquatic fungi. The species, which are found in water as conidia and grow in terrestrial conditions, are referred to as the "water-carried fungi".

We decided to supplement our previous studies on the conidial fungi encountered in the open waters of north-eastern Poland $(\mathrm{C} \mathrm{z} \mathrm{e} \mathrm{c} \mathrm{z} \mathrm{u} \mathrm{g} \mathrm{a} \mathrm{and}$ Or lowska 1993, 1996) with data concerning atypical water forms such as rain water flowing from yarious trees or roof types, and melting snow and ice. The investigations carried out in such atypical niches as morning dew or water extracted from moss Sphagnum and others $(\mathrm{C} \mathrm{z}$ e c z u g a and O r l o w s k a 1994, 1995, 1997a, b) encouraged us to perform further studies of Hyphomycetes species composition in these and other atypical ecological niches. 


\section{MATERIAL AND METHODS}

The studies were carried out from May 1992 to September 1994. The rain water draining during a driving rain from 10 coniferous tree species and 10 deciduous tree species was examined. In the coniferous group Abies concolor (1), Juniperus communis (3), Picea abies (6), Methasequoia glyptostroboides (5), Chamaecyparis pisifera cyanoviridis (2), Pinus sylvestris (8), Pinus strobus (7), Juniperus sabina (4), Thuja orientalis (10), Taxus baccata (9), and in the deciduous group Alnus glutinosa (13), Betula verrucosa (14), Populus nigra (17), Buxus sempervirens (15), Fraxinus excelsior (16), Aesculus hippocastanum (12), Acer platanoides (11), Quercus robur (18), Tilia cordata (20) and Salix alba (19) were examined. Just before the driving rain, a sterilized dish $(40 \times 30 \mathrm{~cm})$ was situated under a tree of the species examined to collect the required amount of rain water draining from a particular tree.

The rain water draining during a storm from six following roofs: sheet copper (A), sheet zinc (B), red tiled (C), asbestic tiled (D), tar paper (E) and thatched roof $(\mathrm{F})$ was also examined. Just before the storm, a sterilized dish $(40 \times 30 \mathrm{~cm})$ was situated under a gutter of the roofs examined to collect the required amount of rain water draining from a respective roofs.

The snow was collected from branches (on the height of $1-1.5 \mathrm{~m}$ ) of three gymnosperm tree species in the Knyszyńska Forest (NE Poland): Pinus sylvestris, Picea abies and Juniperus communis. The required amount of snow was shaken from several trees of each species to a sterilized dish $(30-40 \mathrm{~cm})$ and then transported to the laboratory. The water obtained was filtered trough a gauze to separate tree needles still present in the water. The water obtained was later poured into previously sterilized litre beakers.

The study investigated the ice of stagnant water basins (ponds) and running water bodies (rivers). In January, ice blocks $(20-25 \mathrm{~cm}$ thick) were collected from water basins to sterile containers. Then, in the laboratory they were rinsed with distilled water three times and transferred to sterile dishes to melt. The water formed from the ice collected from each type of water reservoir was distributed to 6 one-litre beakers. Eighteen parameters of this water were determined (Table 1) according to the generally accepted methods ( $\mathrm{Gol}$ terman and Cly mo 1969). Temperature in the laboratory was maintained at $1-2^{\circ} \mathrm{C}$.

For the determinations of the presence of conidia of Hyphomycetes species in the rain water, snow water and ice water, the following procedure was employed: one part of each sample of water was cxamined according to $\mathrm{And} \mathrm{o}$ and $\mathrm{Tubaki}$ (1984a), second part according to $\mathrm{Cz} \mathrm{e} \mathrm{c} \mathrm{z} \mathrm{u} \mathrm{g} \mathrm{a}$ and Or lowska (1993) using cellophane and snake exuviae as bait.

The baits were cut into small pieces and boiled several times with the water being changed each time. They were placed in the containers 
T a ble 1

Chemical composition of the water from investigated water bodics (in $\mathrm{mg} \mathrm{l}^{-1}$ ) $(\mathrm{n}=5$ )

\begin{tabular}{|c|c|c|c|c|}
\hline \multirow{2}{*}{ Parameter } & \multicolumn{2}{|c|}{ Pond } & \multicolumn{2}{|c|}{ River } \\
\hline & Fosa & Dojlidy & Biala & Suprasi \\
\hline Temperature ${ }^{\circ} \mathrm{C}$ & 1.0 & 0.0 & 2.0 & 0.5 \\
\hline $\mathrm{pH}$ & 7.00 & 7.50 & 7.31 & 7.36 \\
\hline $\mathrm{O}_{2}$ & 1.82 & 13.60 & 7.04 & 11.88 \\
\hline COD & 15.09 & 9.38 & 9.02 & 8.80 \\
\hline $\mathrm{CO}_{2}$ & 22.0 & 4.4 & 15.4 & 6.6 \\
\hline Alkalinity in $\mathrm{CaCO}_{3}\left(\right.$ mval $\left.1^{-1}\right)$ & 5.5 & 3.0 & 4.7 & 4.0 \\
\hline $\mathrm{N}-\mathrm{NH}_{3}$ & 0.854 & 0.280 & 0.642 & 0.324 \\
\hline $\mathrm{N}-\mathrm{NO}_{2}$ & 0.008 & 0.014 & 0.011 & 0.012 \\
\hline $\mathrm{N}-\mathrm{NO}_{3}$ & 0.05 & 0.03 & 0.05 & 0.06 \\
\hline $\mathrm{PO}_{4}$ & 3.590 & 0.007 & 1.504 & 1.108 \\
\hline $\mathrm{Cl}$ & 44.0 & 26.0 & 40.0 & 18.0 \\
\hline Total hardness in $\mathrm{Ca}$ & 79.20 & 56.88 & 92.16 & 68.42 \\
\hline Total hardness in $\mathrm{Mg}$ & 26.23 & 13.76 & 22.34 & 14.10 \\
\hline $\mathrm{SO}_{4}$ & 23.04 & 38.26 & 68.70 & 30.85 \\
\hline $\mathrm{Fe}$ & 1.05 & 0.72 & 0.90 & 1.02 \\
\hline Dry residue & 429.0 & 224.0 & 532.0 & 236.0 \\
\hline Dissolved solids & 370.0 & 117.0 & 496.0 & 199.0 \\
\hline Suspended solids & 59.0 & 107.0 & 36.0 & 37.0 \\
\hline
\end{tabular}

with investigated water types. During a 1-mo-period, any kind of cluster from a beaker bottom, side walls and the surface film of the water was examined under a light microscope. Identification of respective species was based on morphology and biometric data of conidiophores and conidia, contained in the keys (Nilsson 1964; Dudka 1974; Ingold 1975; C a rm ic ha e 1 et al. 1980; B r åth e n 1984; M a t s u s h im a 1993) and in the works of the authors who were the first to describe a respective species.

\section{RESULTS}

We found 146 species belonging to Hyphomycetes. Fifty seven taxa were determined in the rain water flowing from 10 coniferous and 10 deciduous tree species, including 17 found in the rain water flowing from both coniferous and deciduous tree species. Sixteen species were found in the water flowing from 
deciduous trees, while 24 in the water from coniferous trees (Table 2). The rain water flowing from different roof types yiclded 33 Hyphomycetes taxa (Table 3). We isolated 26 species of fungi imperfecti from the melting snow collected from 3 coniferous trees (Pinus, Picea, Juniperus) in the Knyszyńska Forest (Table 4). A total of 111 taxa belonging to Hyphomycetes were found in the ice water from 4 water basins ( 2 ponds and 2 rivers) during 2 winter seasons (Table 5).

Fifty five Hyphomycetes species are new to Poland, including 14 species detected in the rain water flowing from trees, 4 from roofs, 6 in melting snow and 31 in ice water.

The above data indicate that a greater number of conidial fungi occur in melting ice water, compared with the some volume of rain water.

Ta ble 2

Hyphomycetes recorded in the rain water falling from trees

\begin{tabular}{|c|c|}
\hline Species of fungi & Species of trees** \\
\hline Acrodictys bambusicola M.B. Ellis & 2,4 \\
\hline Acrodictys elaeidicola M.B. Ellis & $2,9,15$ \\
\hline Acrodictys similis HoL.Jech. & 10 \\
\hline Actinocladium amazonicum Matsushima & 15 \\
\hline Articulospora prolifera Roldan et van der Merve & 15 \\
\hline - Beverwykella cerebriformis Nawawi et Kuthubuth. & $1,6,7,11,16,17,19$ \\
\hline Beverwykella pulmonaria (van Beverwijk) Tubaki & $2,9,15$ \\
\hline Berkleasmium pulchrum Hol.-Jech. et Merc. & 5 \\
\hline Blodgettia indica Subramanian & 19 \\
\hline + Brachiosphaera jamaicensis (Crane et Dumont) Nawawi & 5 \\
\hline Canalisporium caribense (Hol.-Jech. et Merc.) Nawawi et Kuthubuth. & $2,4,9,10$ \\
\hline Canalisparium pulchrum Nawawi et Kuthubuth. & 13 \\
\hline Ceratosporium comutum Matsushima & $9,15,17,18$ \\
\hline Clathrosporium intricatum Nawawi et Kuthubuth. & $1,12,14,17,18,20$ \\
\hline Clathrosparium zalewskii van Beverwijk & 19 \\
\hline Collspora elongata Marvanová & 2,15 \\
\hline Corynespora cubensis Hol.Jech. & 11,17 \\
\hline * Corynesporella superioramifera Matsushima & 4,5 \\
\hline Cunicispora ombrogena Ando et Tubaki & 14,16 \\
\hline * Cylindrocarpon aequatoriale Matsushima & 4 \\
\hline Dactylaria lunata Tzean et Chen & 10 \\
\hline Dicranidion fissile Ando et Tubaki & 6,11 \\
\hline - Endophragmiella quadriloctlaris Matsushima & 4 \\
\hline
\end{tabular}




\begin{tabular}{|c|c|}
\hline Helicoon macrosporum van der Aa ct Samson & 9 \\
\hline Heliscus lugdunensis Sacc. et Therry & $2,6,7,10,11,12,14,16,20$ \\
\hline Hyaloscypha lignicola Abdullah et Webster & 19 \\
\hline Kylindria peruamazonensis Matsushima & 5 \\
\hline Massarina fronsisubmersa Hyde & S \\
\hline * Microsiella pluvioriensis Ando et Tubaki & 14 \\
\hline Mirandina brevispora Matsushima & 17 \\
\hline Mirandina corticola G. Amaud & 8 \\
\hline Ordus tribrachiatus Ando et Tubaki & 18,20 \\
\hline * Paracryptophiale kamaruddinï Kuthubuth. et Nawawi & 4 \\
\hline Pithomyces obscuriseptatus Matsushima & 2,4 \\
\hline Pleuropedium tricladioides Marvanová et Iqbal & 4,9 \\
\hline Pleurophragmium parvisporum (Preuss) Hol-Jech. & 3 \\
\hline Pseudaegerita matsushimae (Matsushima) Webster & 6 \\
\hline Pseudaegerita viridis (Bayl Elliot) Abdullah et Webster & 7 \\
\hline Scolecobasidium variabile Barron et Busch & $2,9,15$ \\
\hline Sigmoidea prolifera (Petersen) Crane & 5 \\
\hline Stagonospora macropycnidia Cunnell & 3,13 \\
\hline Sterigmatobotrys uniseptata Chang & 5,13 \\
\hline Tetracladium maxilliforme (Rostr.) Ing. & 2,4 \\
\hline Tetraploa aristata Berk et Br. & 18 \\
\hline Titaea clarkeae Ellis et Everh. & $1,6,7,11,12,14,16,18,20$ \\
\hline Tricellula aquatica Webster & 10 \\
\hline Tricelbula inaequalis van Beverwijk & 4 \\
\hline Tricladiella piuvialis Ando ct Tubaki & $7,14,16,17,18,20$ \\
\hline Trifurcospora irregularis (Matsushima) Ando et Tubaki & $1,11,16,20$ \\
\hline Tripospermuen canelopardus Ing. Dann et McDougall & $3,7,8,13$ \\
\hline Tripospermum gardineri S. Hughes & 19 \\
\hline Tripospermum infalcatum Ando et Tubaki & 12 \\
\hline Tripospermum myrti (Lind) S. Hughes & 15 \\
\hline Tripospermum porosporiferum Matsushima & 15 \\
\hline Veramyces manuensis Matsushima & 2 \\
\hline Volucrispora aurantiaca Haskins & 2 \\
\hline * Wiesneriamyces conjunciosporus Kuthubuth. et Nawawi & 8,13 \\
\hline
\end{tabular}

* were recorded for the first time from Poland; ** see Material and Methods 


\section{Table 3}

Hyphomycetes found in the rain water falling from six roof types

\begin{tabular}{|c|c|c|c|c|c|c|}
\hline \multirow{2}{*}{ Species of fungi } & \multicolumn{6}{|c|}{ Roof type** } \\
\hline & A & B & C & D & E & $\mathbf{F}$ \\
\hline Acrodictys similis Hol-Jech. & $x$ & & & & & $x$ \\
\hline Actinospora megalospora Ing. & & & & & $x$ & \\
\hline Angulospora aquatica S. Nilss. & & $\mathrm{x}$ & & $\mathrm{x}$ & $x$ & \\
\hline Arborispora palma Ando & & & $\mathrm{x}$ & & & \\
\hline Arbusculina irregularis (Petersen) Marv. et Descals & $x$ & & $\mathrm{x}$ & $\mathrm{x}$ & $\mathrm{x}$ & \\
\hline Beverwykella pulmonaria (van Beverwijk) Tubaki & $\mathrm{x}$ & & $\mathrm{x}$ & & $\mathrm{x}$ & \\
\hline $\begin{array}{l}\text { Canalisporium cariberse (Hol.-Jech. et Merc.) Nawawi } \\
\text { et Kuthubuth. }\end{array}$ & $x$ & $x$ & $x$ & $x$ & $x$ & $x$ \\
\hline Catenomycopsis rosea Constant. & $x$ & & $x$ & & & \\
\hline Clavariena aquatica Nawawi & $x$ & & $\mathrm{x}$ & & & $x$ \\
\hline Colispora elongata Marvanová & & & $x$ & & & \\
\hline Corynespora cubensis Hol.-Jech. & & & $\mathbf{x}$ & & & \\
\hline Corynesporella superioramifera Matsushima & & $x$ & & $x$ & & \\
\hline - Dactylaria lunaia Tzean et Chen & & & & & & $x$ \\
\hline Gyocrffyella tricapillata (Ing.) Marvanová & & & $\mathrm{x}$ & & & \\
\hline Heliscus lugdunensis Sacc. et Therry & $x$ & & & $x$ & & \\
\hline Hyaloscypha lingicola Abdul. et Webster & $x$ & & $x$ & & & \\
\hline - Hyaloscypha zalewskii Desc. et Webster & & & & & & $x$ \\
\hline Lunulospora curvula Ing. & & & & & & $x$ \\
\hline Mirandina corticola G. Amaud & & & & $\mathrm{x}$ & & \\
\hline Neta patuxentica Shearer et Crane & & & $x$ & & & \\
\hline Ordus tribrachiatus Ando et Tubaki & $\mathrm{x}$ & & & & & \\
\hline Pithomyces obscuriseptatus Matsushima & & $x$ & & & & \\
\hline - Scolecobasidium flagelliferum Matsushima & & & & $\mathrm{x}$ & & \\
\hline Speiropsis pedatospora Tubaki & $\mathbf{x}$ & $x$ & & $\mathrm{x}$ & $\mathrm{x}$ & \\
\hline Sporidesmiella hyalosperma (Corda) Kirk & $\mathrm{x}$ & & & & & \\
\hline Titaea clarkeae Ellis et Everh. & & & & $\mathrm{x}$ & $\mathrm{x}$ & \\
\hline Tricellula aquatica Webster & $\mathrm{x}$ & & & & & \\
\hline Tricelulla inaequalis van Beverwijk & $x$ & $x$ & & & $\mathbf{x}$ & \\
\hline Tricladium procerum Marvanová & & & & & $\mathrm{x}$ & \\
\hline Trinacrium subtile Riess & & & $\mathbf{x}$ & & & $\mathrm{x}$ \\
\hline Tripospermum infalcatum Ando et Tubaki & $x$ & & & & & \\
\hline Veramyces manuensis Matsushima & & $x$ & & $x$ & $x$ & \\
\hline * Wiesneriomyces laurinus (Tassi) Kirk & $x$ & & & & & \\
\hline Number of species & 15 & 7 & 12 & 10 & 10 & 7 \\
\hline
\end{tabular}

- were recorded for the first time from Poland; ** see Material and Methods 
In the rain water draining from of twenty tree species the presence of 14 species of aero-aquatic fungi was reported as new to Poland (Table 2). We observed the development of Baverwykella cerebriformis in the rain water draining from California fir, maple, ash, spruce, Weymouth pine and poplar. The species was reported on leaves submerged in water on Malaysia territory by $\mathrm{Naw}$ a w i and $\mathrm{Kuthubuth}$ een (1988). We observed conidia of Brachiosphaera jamaicensis in the rain water draining from dawn redwood. For the first time it was isolated by $\mathrm{Cr}$ a n e and $\mathrm{Dum}$ o n $\mathrm{t}$ (1975) from decaying wood as Actinospora jamaicensis in West Indies. $\mathrm{N}$ a w a w i (D e s c a ls et al. 1976) introduced the name Brachiosphaera jamaicensis. Some of the hyphomycete species, which appear new to Poland, were first described from the river-basin of the Amazon by M a t s u s h i m a (1993). The growth of such species as Corynesporella superioramifera, Endophragmiella quadrilocularis and Tripospermum porosporiferum was observed by M a t s u s h i a (1993) on putrescent leaves of latifolious plant in land conditions in the Cuyabeno National Park in Ecuador, while Veromyces manuensis on putrescent palm petioles in the Manu National Park in Peru. Such species as Cylindrocarpon aequatoriale, Kylindria peruamazonensis and Pithomyces obscuriseptatus werc found on putrescent palm petioles in the Amazon tributaries - the Rio Mamon and the Rio Negro (Peru), and the Rio Yuturi (Equador). Microstella pluvioriens was first isolated from the rain water flowing from Metasequoia glyptostroboides in Japan by A $\mathrm{n}$ do and T u b a k i (1984b).

In our studies Paracryptophiale kamaruddinii occurred only in the rain water draining from savin juniper. It was first isolated by $\mathrm{Ku} \mathrm{th} u$ b u thee $\mathrm{n}$ and $\mathrm{N}$ a w a w i (1994) from decaying litter submerged in a freshwater stream in Malaysia. Pseudaegerita matsushimae was observed in the rain water draining from spruce only. It was first recorded by $\mathrm{M}$ a $\mathrm{t}$ s u sh i m a (1975) as Papulaspora viridis on a decaying Quercus sp. leaf, Kumamoto, Japan. A b d u $11 \mathrm{a} \mathrm{h}$ and W e b s ter (1983) observed the development of this fungus also in Quercus sp. leaf in Scotland, introducing the name Pseudaegerita matsushimae. We observed the growth of Tricladiella pluvialis in the rain water draining from Weymouth pine, poplar, durmast, lime, ash and birch. It was reported by An do and T u baki (1984b) in the rain water draining from leaves of Quercus acutissima in Japan. This is the second site of this fungus. Wiesneriomyces conjunctosporus in our case grew only in the rain water draining from pine and alder. It was first found by Kuthubutheen and $\mathrm{Nawawi}$ (1994) on decaying submerged leaves in Malaysia.

In the rain water falling from six roof types such species as Dactylaria lunata, Hyaloscypha zalewskii, Scolecobasidium flagelliferum and Wiesenriomyces laurinus are new to Poland. We observed Dactylaria lumata in the rain water falling from a thatched roof, this fungus was first described from fallen parts of twigs in a recreation forest in Taiwan ( $\mathrm{z} \mathrm{c}$ a $\mathrm{n}$ and $\mathrm{C} \mathrm{h} \in \mathrm{n}$ 1991). 
Table 4

Hyphomycetes found in snow water

\begin{tabular}{|c|c|c|c|}
\hline Species of fungi & $\begin{array}{l}\text { Pinus } \\
\text { sylvestris }\end{array}$ & $\begin{array}{l}\text { Picea } \\
\text { abies }\end{array}$ & $\begin{array}{l}\text { Juniperus } \\
\text { communis }\end{array}$ \\
\hline Acradictys bambusicola M.B. Ellis & $\mathrm{x}$ & $\mathrm{x}$ & $\mathrm{x}$ \\
\hline Acrodictys eloeidicola M.B Ellis & & & $\mathrm{x}$ \\
\hline Anguillospora pseudolongissima Ranzoni & & $\mathrm{x}$ & $\mathrm{x}$ \\
\hline Arborispora palma Ando & & & $\mathrm{x}$ \\
\hline Arbusculina irregularis (Petersen) Marv. ct Descals & & $\mathrm{x}$ & \\
\hline Calcarispora hiemalis Marvanová et Marvan & $\mathrm{x}$ & $x$ & \\
\hline * Camposporium pellucidum (Grove) S. Hughes & & $\mathrm{x}$ & \\
\hline $\begin{array}{l}\text { Canalisporitum caribense (Hol.-Jech. et Merc.) Nawawi } \\
\text { et Kuthubuth. }\end{array}$ & $\mathrm{x}$ & $x$ & \\
\hline $\begin{array}{l}\text { Canalispora puichrum (Hol_-Jech. et Merc) Nawawi et } \\
\text { Kuthubuth. }\end{array}$ & & & $\mathrm{x}$ \\
\hline Ceratosporitum cornutum Matsushima & & $\mathrm{x}$ & \\
\hline Clathrosporium intricatum Nawawi et Kuthubuth. & & & $\mathrm{x}$ \\
\hline Corynesporella simpliphora Matsushima & $\mathrm{x}$ & $\mathrm{x}$ & \\
\hline Mirandina corticola G. Arnaud & $\mathrm{x}$ & $\mathrm{x}$ & $\mathrm{x}$ \\
\hline * Monodictys peruviana Matsushima & & $\mathrm{x}$ & \\
\hline Mycocentrospora aquatica (Iqbal) Iqbal & $x$ & & \\
\hline Paracryptophiale kamaruddinii Kuthubuth et Nawawi & $\mathbf{x}$ & & \\
\hline Paraepicoccum amazonense Matsushima & & & $\mathrm{x}$ \\
\hline *Polystratorictus fusarioideus Matsushima & $\mathrm{x}$ & $\mathrm{x}$ & \\
\hline Pseudaegerite corticalis (Peck) Crane et Schok. & & & $\mathrm{x}$ \\
\hline${ }^{*}$ Sporidesmium moniliforme Matsushima & $x$ & & $\mathrm{x}$ \\
\hline Trifurcospora irregularis (Matsush.) Ando et Tubaki & & & $\mathrm{x}$ \\
\hline Trinacrium subtile Riess & & $\mathrm{x}$ & \\
\hline - Tripospermum acerinum (Sydow) Spegazzini & $\mathrm{x}$ & $\mathrm{x}$ & \\
\hline Tripospermum myrti (Lind) S. Hughes & & $\mathrm{x}$ & $\mathrm{x}$ \\
\hline Vargamyces aquaticus (Dudka) Tóth & $\mathbf{x}$ & $\mathrm{x}$ & \\
\hline *Veronaea botryosa Cif et Montemartini & $\mathbf{x}$ & & \\
\hline Number of species & 12 & 15 & 12 \\
\hline
\end{tabular}

* were recorded for the first time from Poland. 
Hyaloscypha zalewskii described in the previous century as Clathrosphaerina spirifera ( $\mathrm{Z}$ a l e w s k i 1888). It turned out to be a holomorphic species, in which Clathrosphaerina zalewskii (van B e v e r w i j k 1951) and Hyaloscypha zalewskii were included (Descals and Webster 1976). We observed the growth of Hyaloscypha zalewskii in the rain water falling already from a thatched roof. Scolecobasidium flagelliferum were found in the rain water falling from asbestic tiled roof and Wiesneriomyces laurinus from a sheet copper roof. Scolecobasidium flagelliferum was first described from decaying petiole in the tributary of the Rio Amazonas ( $M$ a t s u s h i m a 1993). Wiesneriomyces laurinus was first described from leaves in a stream in Malaysia (K u thubutheen and $\mathrm{N}$ aw a w i 1988), while the earlier encountered at several different sites ( $\mathrm{K}$ i r k 1984), mostly on fallen leaves.

Monodictys peruviana and Polystratorictus fusarioideus, new to the Polish mycoflora, were first reported from terrestrial conditions on palm-petolio putrescenti in the valley of the Rio Amazonas by M a t s u s h i m a (1993). On the same substrate in the water of the Rio Negro and Rio Yalapa another species - Sporidesmium moniliforme was found ( $\mathrm{M}$ a $\mathrm{ts} \mathrm{u} \mathrm{s} \mathrm{h} \mathrm{i} \mathrm{m} \mathrm{a} \mathrm{1993).}$ Composporium pellucidum has been long known as a species of terrestrial ( $\mathrm{H} \mathrm{u} \mathrm{g} \mathrm{h} \mathrm{es} \mathrm{1951)} \mathrm{and} \mathrm{aquatic} \mathrm{conditions} \mathrm{(} \mathrm{T}$ ó $\mathrm{h} \mathrm{1973;} \mathrm{I} \mathrm{n} \mathrm{g} \mathrm{o} \mathrm{ld} \mathrm{1975).}$ Later it was found in Hungary on forest litter ( $\mathrm{G}$ ó n c z öl and R é va y 1983). Another species new to Poland, Tripospermum acerinum, has been already encountered in terrestrial conditions (A n d o 1992) and described as Triposporium acerinum (S y d ow 1902). Later S p e g a z z in i (1918) erected a new genus, Tripospermum and created a new combination, Tripospermum acerinum. Verona botryosa was first described by $\mathrm{C}$ if e r $\mathrm{r}$ i and Montemartin i (1957) in Italy, then it was reported as Sympodina caprophila by Subramanian and Lod ha (1964). M a t s u sh i m a (1971) called this fungus Scolecobasidium coprophilum, although in a monograph on Hyphomycetes of the water basin of the Amazon he gave it the name suggested by $\mathrm{Ciferri}$ and $\mathrm{M}$ ontemartini (1957). In the water basin of the Amazon, this fungus was found on fallen palma petioles both in the Rio Negro and Tambopata reserve (M a t s us h i m a 1993).

New Hyphomycetes species, such as Acrodictys peruamazonensis, Cylindrocarpon aequatoriale, Fusticeps laevisporus, Helminthosporium bigenum, Menispora amazonensis and Paradactylella peruviana were first described by $\mathrm{M}$ a ts u s h i m a (1993) from the river basin of the Amazon. Paradactylella peruviana was found on petiole in the reserve of Tambopata in Peru, while the other species in the Amazon tributaries, mainly on putrescent palm petioles. Acrodictys martimii was first noted in West Indies by $\mathrm{Cr}$ a n e and $\mathrm{D} \mathrm{u}$ m o n t (1975). Also M a t s u s h i m a (1993) observed the growth of this fungus on fallen twigs in the Rio Ampiyach. Taeniolina deightonii was described by $\mathrm{Crane}$ and $\mathrm{Schoknecht}$ (1981), when revising Torula species collected from Vismia guineensis in Sierra Leone by F.C. Deighton. Pseudospiropes subliferus has been known since the first half of the previous century as 
Table 5

Hyphomycetes found in the water from melting ice

\begin{tabular}{|c|c|c|c|c|}
\hline \multirow{2}{*}{ Species of fungi } & \multicolumn{2}{|c|}{ Pond } & \multicolumn{2}{|c|}{ River } \\
\hline & Fosa & Dojlidy & Biała & Supras! \\
\hline Acrodictys bambusicola M.B. Ellis & $\mathrm{x}$ & $\mathrm{x}$ & $x$ & $x$ \\
\hline Acrodictys elaeidicola M.B. Ellis & & $\mathrm{x}$ & & $\mathrm{x}$ \\
\hline - Acrodictys martinï Ceane et Dumont & $x$ & $\mathbf{x}$ & $x$ & $\mathrm{x}$ \\
\hline *Acrodictys peruamazonensis Matsushima & $x$ & & & \\
\hline Actinospora megalospora Ing. & & $\mathrm{x}$ & & \\
\hline Alatospora acuminata Ing. & $x$ & $\mathrm{x}$ & & $\mathrm{x}$ \\
\hline Alatosessilispora bibrachiata Ando et Tubaki & & & $x$ & \\
\hline Anguillospora crassa Ing & & & & $\mathrm{x}$ \\
\hline Anguillospora longissima (Sacc. et Syd.) Ing. & $\mathrm{x}$ & $\mathrm{x}$ & $\mathrm{x}$ & $\mathrm{x}$ \\
\hline Anguillospora pseudolongissima Ranz. & $x$ & $x$ & & \\
\hline Angulospora aquatica $\mathbf{S}$. Nilss. & $x$ & $\mathrm{x}$ & $\mathrm{x}$ & $\mathrm{x}$ \\
\hline Arborispara multisurcularis Ando & & & $x$ & $\mathrm{x}$ \\
\hline Arbusculina fragmentans Marvanová et Descals & $\mathrm{x}$ & $\mathrm{x}$ & $\mathrm{x}$ & $\mathrm{x}$ \\
\hline * Beltraniella peruamazonica Matsushina & $\mathrm{x}$ & & & \\
\hline Blodgettia borneti Wright & & & & $x$ \\
\hline Bladgettia indica Subramanian & $\mathrm{x}$ & & & \\
\hline Calcarispora hiemalis Marvanová et Marvan & & & $x$ & \\
\hline Camposporium pellucidum (Grove) S. Hughes & $\mathbf{x}$ & $\mathrm{x}$ & & \\
\hline $\begin{array}{l}\text { Canalisporium caribense (Hol.-Jech. et Merc.) Nawawi } \\
\text { et Kuthub. }\end{array}$ & $\mathrm{x}$ & $\mathrm{x}$ & $\mathbf{x}$ & $x$ \\
\hline Canalisporium pulchrum (Grove) Hughes & & & $\mathbf{x}$ & \\
\hline Centrospora aquatica Iqbal & $\mathrm{x}$ & & $\mathrm{x}$ & $x$ \\
\hline Centrospora filiformis (Greath) Petersen & & & $\mathrm{x}$ & \\
\hline Clavariopsis aquatica de Wild. & & $\mathrm{x}$ & & $x$ \\
\hline - Clavariopsis azlanii Nawawi & & & & $x$ \\
\hline Clavatospora longibrachiata (Ing.) S. Nilss. & & $x$ & & $\mathrm{x}$ \\
\hline Clavatospora stellata (Ing. et Cox) S. Nilss. & & & $\mathrm{x}$ & \\
\hline Clavatospora tentacula (Umphlett) S. Nilss. & $\mathrm{x}$ & & & $\mathrm{x}$ \\
\hline Clavariana aquatica Nawawi & & $x$ & & $x$ \\
\hline Colispora elongata Marvanová & $\mathrm{x}$ & & & $\mathrm{x}$ \\
\hline Condylospora spumigena Nawawi & & & & $\mathrm{x}$ \\
\hline Corynespora cubensis Hol.-Jech. & & $\mathrm{x}$ & $\mathrm{x}$ & \\
\hline
\end{tabular}




\begin{tabular}{|c|c|c|c|c|}
\hline Corynesporella simpliphora Matsushima & $x$ & & $\mathrm{x}$ & \\
\hline Corynesporella superioramifera Matsushima & $\mathrm{x}$ & $\mathrm{x}$ & $\mathrm{x}$ & \\
\hline *Cylindrocarpon aequatoriale Matsushima & $\mathrm{x}$ & $\mathrm{x}$ & & $x$ \\
\hline Dactylella submersa (Ing.) S. Nilss. & $\mathrm{x}$ & $\mathrm{x}$ & & $x$ \\
\hline Descalsia cruclaia Roldan et Honrubia & & & $\mathrm{x}$ & \\
\hline Dimorphospora follicola Tubaki & $x$ & & & \\
\hline * Diplocladiella appendiculata Nawawi & $\mathrm{x}$ & & & $x$ \\
\hline Flagellospora stricta S. Nilss. & & & & $x$ \\
\hline Fusarium candida Matsushima & & $x$ & & $x$ \\
\hline Fusarium aquaeductum (Radlk. et Rabb.) Lagh. & & & $\mathrm{x}$ & $\mathrm{x}$ \\
\hline Fusticeps laevisporus Matsushima & & & & $x$ \\
\hline Gyoerffyella myrmecophagiformis Melnik et Dudka & & & $\mathrm{x}$ & $\mathrm{x}$ \\
\hline Helicoon pluriseptatum van Beverwijk & & & & $\mathrm{x}$ \\
\hline "Helicoma vaccinia Carris & & & & $x$ \\
\hline Heliscus lugdunensis Sace. et Therry & $\mathrm{x}$ & $\mathrm{x}$ & $\mathbf{x}$ & $x$ \\
\hline Heliscus submerstus Hudson & & $x$ & & \\
\hline Helminthosporium bigenum Matsushima & $\mathrm{x}$ & & & \\
\hline *Isthmolongtspora geniculata Nawawi et Kuthubuth. & & $\mathrm{x}$ & & $x$ \\
\hline Kontospora halophila Roldan et Honrubia & $\mathbf{x}$ & & & $x$ \\
\hline * Lateriramulasa unänflata Matsushima & & & & $\mathrm{x}$ \\
\hline Lemonniera aquatica de Wild. & $\mathbf{x}$ & $\mathrm{x}$ & $x$ & $x$ \\
\hline - Lemonriera centrosphaera Marvanová & $\mathrm{x}$ & & & \\
\hline Lemonniera filiforme Petersen & & $\mathrm{x}$ & & \\
\hline Lemonniera terrestris Tubaki & & $\mathrm{x}$ & & $x$ \\
\hline Lumulospora curvula Ing. & $\mathrm{x}$ & $x$ & $\mathrm{x}$ & $\mathrm{x}$ \\
\hline Margaritispora aquatica Ing. & & $x$ & & \\
\hline - Menispora amazonensis Matsushima & & & $\mathrm{x}$ & \\
\hline Microstella pluvioriens Ando et Tubaki & & $x$ & & \\
\hline Mirandina corticola G. Arnaud & $\mathrm{x}$ & $\mathrm{x}$ & $\mathrm{x}$ & $\mathrm{x}$ \\
\hline Monodictys peruviana Matsushima & $\mathrm{x}$ & $\mathrm{x}$ & & \\
\hline Monolosporella microaquatica (Tubaki) S. Nilss. & & & & x \\
\hline - Nectria favo-lanata Berkeley et Broome & & & & $\mathrm{x}$ \\
\hline - Paraarthrocladium amazonense Matsushima & & & & $\mathrm{x}$ \\
\hline - Paradactylella peruviana Matsushima & & $\mathrm{x}$ & $\mathrm{x}$ & \\
\hline Paraepicoccum amazonense Matsushima & & $\mathrm{x}$ & & \\
\hline
\end{tabular}


Tab. 5 cont.

Species of fungi

\begin{tabular}{|c|c|c|c|c|}
\hline \multirow{2}{*}{ Species of fungi } & \multicolumn{2}{|c|}{ Pond } & \multicolumn{2}{|c|}{ River } \\
\hline & Fosa & Dojlidy & Biała & Suprasi \\
\hline * Phaeodactylium acutisporum Matsushima & & $\mathrm{x}$ & & \\
\hline - Phialogeniculata multiseptata Matsushima & $\mathrm{x}$ & & & \\
\hline Piricauda cubensis Hol. Jech. ct Merc. & & $\mathrm{x}$ & $\mathrm{x}$ & $\mathrm{x}$ \\
\hline Pithomyces obscuriseptatus Matsushima & & $\mathrm{x}$ & $x$ & \\
\hline Polycladium equiseti Ing. & & $\mathrm{x}$ & & \\
\hline Polystratorictus fusarioideus Matsushima & $\mathbf{x}$ & $\mathrm{x}$ & & $\mathrm{x}$ \\
\hline Pseudaegerita corticalis (Peck) Crane et Schak. & & & $\mathrm{x}$ & $\mathrm{x}$ \\
\hline Pseudaegerila viridis (Bay! Elliot) Abdullah et Webster & & & & $\mathrm{x}$ \\
\hline *Pseudohansfordia dimorpha Matsushima & $\mathrm{x}$ & $\mathrm{x}$ & & \\
\hline *Pseudospiropes lotorus Morgan-Jones & & $\mathrm{x}$ & & $\mathrm{x}$ \\
\hline *Psendospiropes subliferus (Corda) M.B. Ellis & & & & $\mathrm{x}$ \\
\hline * Pyricularia peruamasonica Matsushima & & $\mathrm{x}$ & & \\
\hline Retiarius bovicornutus Olivier & & & & $\mathrm{x}$ \\
\hline Scolecabasidium flagelliferum Matsushima & $x$ & & & \\
\hline * Scolecobasidium fusarioideum Matsushima & $\mathrm{x}$ & & & \\
\hline * Scolecosporiella amazonensis Matsushima & $\mathrm{x}$ & & & \\
\hline Stachybotris theobromae Hansf. & & & & $\mathrm{x}$ \\
\hline - Stemphyliomma tambopataense Matsushima & & & & $\mathrm{x}$ \\
\hline - Stemphyliomma terricola Mon. Chary et Ram. & $\mathrm{x}$ & & $x$ & $\mathrm{x}$ \\
\hline Sterigmatobotris witiseptata Chang & & & $\mathrm{x}$ & $\mathrm{x}$ \\
\hline - Taeniolina deightonii Crane et Schaknecht & & $x$ & & $\mathbf{x}$ \\
\hline Teirachaetum elegans Ing. & & & & $\mathrm{x}$ \\
\hline Tetracladium marchalianum de Wild. & $\mathrm{x}$ & $\mathrm{x}$ & $x$ & $\mathbf{x}$ \\
\hline Teiracladium maxilliformis (Rostr.) Ing. & $\mathrm{x}$ & $\mathrm{x}$ & $\mathrm{x}$ & $\mathrm{x}$ \\
\hline Tetracladium setigerum (Grove) Ing. & $x$ & $\mathrm{x}$ & $\mathrm{x}$ & $x$ \\
\hline Tricellula aquatica Webster & $\mathrm{x}$ & $\mathrm{x}$ & $\mathrm{x}$ & $\mathrm{x}$ \\
\hline Tricellula inaequalis van Beverwijk & & $\mathrm{x}$ & & \\
\hline Tricladiam angulatum Tubaki & & $\mathrm{x}$ & & $\mathrm{x}$ \\
\hline Tricladium anomalum Ing. & & & $\mathrm{x}$ & \\
\hline Tricladium gracile Ing. & $x$ & & $\mathrm{x}$ & \\
\hline Tricladium marylandicum Crane & $\mathrm{x}$ & $x$ & & \\
\hline Tridentaria sp. & & & & $\mathrm{x}$ \\
\hline
\end{tabular}




\begin{tabular}{|l|c|c|c|c|}
\hline Trifurcospora irregularis (Matsushima) Ando et Tubaki & & & $\mathrm{x}$ \\
\hline Trinacrium subtile Riess & $\mathrm{x}$ & $\mathrm{x}$ & $\mathrm{x}$ & $\mathrm{x}$ \\
\hline Tripospermun infalcatum Ando et Tubaki & & & & $\mathrm{x}$ \\
\hline Tripospermum myrti (Lind) Hughes & $\mathrm{x}$ & & $\mathrm{x}$ \\
\hline Tripospermum pes-gallinae Cif. Bat. et Nasum & & $\mathrm{x}$ & $\mathrm{x}$ \\
\hline Tripospermum prolongatum Sindair et Morgan-Jones & & & $\mathrm{x}$ & \\
\hline Triscelophorus monosporus Ing. & & & $\mathrm{x}$ & $\mathrm{x}$ \\
\hline -Triscelophorus magnificus Petersen & & $\mathrm{x}$ & & \\
\hline Varicasporium delicatum Iqbal & & $\mathrm{x}$ & & \\
\hline Varicasporium elodece Kegel & & $\mathrm{x}$ & & \\
\hline Veronaea botryosa Cif. et Montemartini & $\mathrm{x}$ & $\mathrm{x}$ & $\mathrm{x}$ & $\mathrm{x}$ \\
\hline Volucrispora aurantica Haskins & 45 & 55 & 41 & 66 \\
\hline Volucrispora graminea Ing et al. & & & \\
\hline Number of species & & & & \\
\hline
\end{tabular}

* were recorded for the first time from Poland

Helminthosporium subuliferum (C o r d a 1837 cited from $\mathrm{M}$ a $\mathrm{s}$ u s h i m a 1993). It was included in a number of genera ( $\mathrm{H} \mathrm{u} \mathrm{g} \mathrm{hes} \mathrm{1958),} \mathrm{to} \mathrm{be} \mathrm{finally}$ classified as the genus Pseudospiropes (E 11 i s 1976). Stemphyliomma terricola was reported by M a o h a a Chary and R a ma r a (1972) from the soil in India. Both of these fungi were observed by $\mathrm{M}$ a $\mathrm{tsu} \mathrm{sh}$ i m a (1993) on fallen palm petioles in the reserve of Tamopata. Nectria flavo-lanata is known as anamorph of Actinostilbe sp. (M a t s u s h i m a 1993). In our study, it was found in the water from ice collected from the Supraśl river.

Also Diplocladiella apendiculata found in the pond Akcent and the Supraśl river is new to Poland. This fungus was first described by $\mathrm{N}$ a w a w i (1987) from twig fragments immersed in the Gombak river in Malaysia. Hellicoma vaccinii, also new to Poland, was first observed by C a r r is (1989) who found it colonizing a vertical scar on a stem section of Vaccinium elliotii collected near the Satilla River, Ware co, Georgia, USA. Its growth was also observed by M a ts us him a (1993) on decaying palm petioles, the Rio Ampiyacu, a tributary of the Amazon in Loreto, Peru. We found it in the ice collected from the Supraśl river. Also Lateriramulosa uniinflata was found in the Supraśl river. This fungus was first described in terrestrial conditions by $\mathrm{M}$ a t s u s im a (1971) from decaying banana leaves on the Solomon Islands. It was also encountered in Europe, e.g. in the litoral zone in water among plant debris in a blind branch of the Svratka river, near Brno and in several other watercourses of the region (M a r v a n o vá 1973). Pseudospiropes lotorus first described by $\mathrm{M}$ o r g a n-J o n es (1973) from roots of Lotus corniculatus in Alabama, USA, in our study was found in the pond Dojlidy and in the Supraśl river. 


\section{DISCUSSION}

In our study most fungi grew in the rain water draining from the coniferous trees. This may suggest that the surfaces of certain coniferous trees act as a substratum for a larger number of species than deciduous trees. This phenomenon could be the resultant of several factors specific to the coniferous species, being a more stable habitat for certain Hyphomycetes, since they do not lose needles every year, but every few years (with the exception of dawn redwood). It is also known that coniferous species secrete sticky resin, which can arrest wind-carried conidia.

The most frequently encountered fungus in the rain water draining from twenty tree species during a driving rain was Heliscus lugdunensis and Titaea clarkeae. Heliscus lugdunensis was noted in northeastern Poland in water of the Supraśl river ( $\mathrm{Czeczuga}$ and O $10 \mathrm{wska}$ 1993), and even in a micropool, temporarily made of melting terrestrial snow ( $\mathrm{C} \mathrm{z} \mathrm{e} \mathrm{c} \mathrm{z} \mathrm{u} \mathrm{g} \mathrm{a}$ 1992); and in the water of Sphagnum (C z e c z u g and O r low s k a 1997b). Earlier this fungus was also found in water conditions in France (M o r e u and M r e a u 1949). The history of the description of this species is of great interest due to, among other things, the environmental factors-dependent dimorphism of its conidia (D u d k a 1985). Heliscus lugdunensis was first described in the previous century as a terrestrial fungus from the pine bark in Italy (S a c c a r d o 1880). I n g o I d (1942), when distinguishing the aquatic Hyphomycetes group, described the aquatic species Heliscus aquaticus as having much in common with the Saccardo's $H$. lugdunensis and $H$. aquaticus and suggested that they were one and the same species $H$. lugdunensis ( $\mathrm{n} \mathrm{g} \mathrm{o} / \mathrm{d}$ and $\mathrm{C} \mathrm{ox} \mathrm{1957).} \mathrm{In} \mathrm{extra-aquatic}$ conditions, Heliscus lugdunensis was, apart from the pine bark, also found in the forest bedding (S a n de r s and W e b s ter 1978) and soil roots of the alder ( $\mathrm{F}$ is h e $\mathrm{r}$ et al. 1991). Also Titaea clarkeae was first described in the previous century (E 11 is and Everhart 1891). This fungus was found on Dichaena quercina (naw known as Polymorphum quercinum) and on Quercus ilicifolia, at Vineland, N.J., U.S.A. S u t t o n (1984) observed the type material of the fungus, and described and illustrated it in detail. However A n do and $\mathrm{Ka} \mathrm{wam} \mathrm{oto} \mathrm{1986)} \mathrm{isolated} \mathrm{this} \mathrm{fungus} \mathrm{from}$ rain water on intact leaves of Carpinus tschonoskii in Tokyo. We also found this species in the water of petmoss in northeastern Poland $(\mathrm{C} \mathrm{z} \mathrm{e} \mathrm{c} \mathrm{z} \mathrm{u} \mathrm{g} \mathrm{a}$ and Orlowsk a 1997b).

Only two species, Canalisporium caribense and Mirandina corticola were found to grow in the water collected from tree, roof, snow and ice. Canalisporium caribense belong to the so called lignicolous group, first described in terrestrial conditions in Cuba $(\mathrm{H} \circ \mathrm{lu}$ b o v á-J e c hov á and M e r c a d o 1984). We have already found this species in morning dew drops on meadow grass ( $\mathrm{Czc}$ c z u g a and O r łow s k a 1997a), in 13 springs of 20 investigated in the Knyszyńska Forest ( $\mathrm{C} \mathrm{z} \mathrm{e} \mathrm{z} \mathrm{u} \mathrm{g} \mathrm{a} \mathrm{and}$ 
O r l o w s a 1996). Mirandina corticola was first described also in terrestrial conditions by A r n a u d (1952). The Gön c z öl and R é v a y (1983) found it in the sediment of several litterleaf samples in Hungary. In northeastern Poland, Mirandina corticola was encountered in the water of several springs of the Knyszyńska Forest $(\mathrm{Czeczug}$ a and $\mathrm{O}$ r 10 w$\mathrm{s} \mathrm{k}$ a 1996), in small forest lakes called suchary in the Augustowska Forest (C z e c z u g a 1995) and coastal water of some lakes in the Suwałki District (C z e c z uga et al. 1997).

The present study reveals that certain Hyphomycetes species can grow on the roofs of buildings covered with different material both in rural and urban areas. The fungi, some of which have other habitats (e.g., tree leaves), and their conidia were arrested to roofs after they had come here with wind. A substrate for most of these species is an organic matter filling up roof unevenness. Short-lasting morning damp and rainwater may help in sporification ( $\mathrm{T} u \mathrm{~b}$ a k i et al. 1985). It can be assumed that not only conidia but also mycelia of these fungi are able to survive dry weather periods. This supposition is substantiated by the data obtained in recent years by $\mathrm{S} r$ idh a $\mathrm{r}$ and $\mathrm{K}$ a veriappa (1988). It was found that aquatic fungi growing on Coffea arabica and Hovea brasiliensis leaves collected from the water of a small stream and then kept in the laboratory under dry conditions for $360 \mathrm{~d}$, continued to grow on being returned to a water environment. These were aquatic fungi of the Hyphomyces genus. The data provide evidence of the mechanisms of adaptation of aquatic fungi to their existence in waters, which during the year periodically dry up. In such cases as soon as the water returns, the fungi begin to develop. Most Hyphomycetes species were found to develop in rainwater samples from sheet copper, the fewest from sheet zinc and thatch roof, which is difficult to interpret explicitly. It may be associated with the size and unevenness degree of roof surface (it can facilitate the arrest of organic matter and conidia). Among the roofs examined sheet copper was the largest and most uneven surface, which was connected with the roof construction, and sheet zinc was the smoothest, while the thatched roof was the smallest. The texture of each roof may be associated also as one factor, which affects the difference in number and flora of inhabiting fungi in each roof. Thus, the number of species found is probably a resultant of all these factors.

As we have already mentioned, the occurrence of phycomycetes in the snow water collected from the ground was studied by many mycologists (K obayasi and Fukushima 1952; T ubaki 1960; Nilss on 1964; C z e c u g a 1992). A comparison of data reported by these authors with our present findings indicates that significant differences occur in the species composition. While the snow water obtained from the ground contents species, which are normally found in water bodies, the samples of the snow water collected from coniferous trees show a predominance of the so-called aero-aquatic hyphomycetes. Among the 26 species found in the snow 
water from coniferous trees only a few belong to the group of aquatic hyphomycetes. How could one explain the presence of hyphomycetes conidia in the snow collected from coniferous tree branches? It seems that conidia of the hyphomycetes species present in the air and carried by the wind fall on coniferous tree needles and branches, attracted by sticky resin, before the trees get covered with snow, i.e. in autumn. The snow, shaken off to dishes, contains conidia. They are also present in the snow water, initiating mycelia, which in turn produce new conidia in laboratory conditions.

Water in the pond Fosa is more trophic than in the pond Dojlidy. Similarly, water of the Biała river, compared to the Supraśl is trophically more abundant (Table 1). This could explain the differences in the number of species found in the respective ponds and rivers. The ice water collected from the pond Dojlidy contained more species than the water in the pond Fosa. Also, the ice water from the Supraśl river had more species than the Biala river. Furthermore, the largest number of Hyphomycetes species in the Suprasil river may be associated with the fact that this river flows through the Knyszyniska Forest, while the Biala river through the town of Bialystok. In the case of ponds, the Fosa is situated in the very centre of Bialystok, while the Dojlidy on the town periphery. According to F a b r e (1996), afforestation has an effect on the number of Hyphomycetes fungi of the particular watercourse. The occurrence of Hyphomycetes spores in ice explains sudden appearance of fungi in sulphur hydrogen stagnant water bodies observed in our studies following ice cover melting. In ice-covered basins, a sudden increase is noted in sulphur hydrogen content in the whole water space. This causes death of all living organisms. We did not manage to culture any Hyphomycetes species, even with the use of different baits, in water samples collected from such water bodies. However, a few days after the ice cover had melted, both aero-aquatic and typically aquatic species appeared in water samples. The presence of aero-aquatic Hyphomycetes could be easily explained by air movements (wild-blown spores). However, sudden appearance of typically aquatic Hyphomycetes species seemed strange. The present study throws some light on the problem. The spores found in ice are isolated from a pernicious effect of sulphur hydrogen, allowing their growth after ice melting. At the present state of knowledge of the biology of aquatic fungi the occurrence of certain rare fungus species in ice-melting water still remains unexplained. This refers not only conidial, but also zoosporic fungi ( $\mathrm{C} \mathrm{z} \mathrm{e} \mathrm{c} \mathrm{z} \mathrm{u} \mathrm{g} \mathrm{a} \mathrm{et} \mathrm{al.}$ 1999). Our long-term studies on zoosporic and conidial fungi carried out in the same water basins from which ice was collected as well as in other reservoirs of northeastern Poland in different seasons of the year, using the same methods and baits, have not revealed the presence of these aquatic fungus species. They were found only in the ice-melting water collected from these basins. Moreover, the number of species observed in the adequate volume of ice-melting water is significantly larger than in the same volume of water collected from the same basin not only in winter but in any other season 
of the year. This may be associated with water properties during freezing and particularly with surface tension, being the highest at water temp. of $0^{\circ}$. According to $\mathrm{R}$ u t t n e r (1962), a specific biotop is formed in the zone of the highest surface tension, with a specific accumulation of organisms, called neuston. As water temperature decreases, the increasing surface tension of the upper layer seems to attract various forms of neuston-forming aquatic fungi from the lower layers. Thus, their highest condensation occurs in the layer of water, which turns into ice. This assumption needs to be confirmed.

According to the keys of aquatic Hyphomycetes (N ils s o n 1964; Ing old 1975; C a rm i chael et al. 1980; B r a then 1984; $\mathrm{D}$ u d k a 1985) 146 species found in the study included all three species of the genus Anguillospora, Angulospora aquatica, Centrospora aquatica, Clavariopsis aquatica, Dactylella submersa, Heliscus lugdunensis, Lemonniera aquatica, Lunulospora curvula, Mycocentrospora aquatica, Palycladium equiseti, Tetrachaetum elegans, 4 species of the genus Tetracladium and Tricladium and Tricellula aquatica. Most species were found in melting snow water. The fewest fungi were encountered in the rain water from trees. Most of these species have been found under fallen leaves in forest basins, in river valleys or at a certain distance from water basins ( $\mathrm{F}$ is h e $\mathrm{r}$ et al. 1991). This would suggest that typical aero-aquatic species can be found in open waters, while aquatic species in terrestrial conditions. The division into aero-aquatic and aquatic $\mathrm{Hyp}$ homycetes is relative, as in both cases water (as morning dew, raindrop or snow) is necessary to form conidia.

\section{REFERENCES}

A bdullah S. K., We bster J. 1983. The aero-aquatic genus Pseudaegerita. Trans. Br. Mycol. Soc. 80: $247-254$.

A n d o K. 1992. A study of terrestrial aquatic hyphomycetes. Trans. Mycol. Soc. Japan 33: $15-425$.

A n d o K, K a w a m o t o I. 1986. Materials for the fungus flora of Japan (41). Trans. Mycol. Soc. Japan 27: $321-326$.

A n d o K., T u b a k i K. 1984a. Some undescribed hyphomyeetes in the rain drops from intact leaf-surface. Trans. Mycol. Soc. Japan 25: 21-37.

A n d o K., T u b a k i K. 1984b. Some undescribed hyphomycetes in rainwater draining from intact trees. Trans. Mycol. Soc. Japan 25: 39-47.

A r a u d G. 1952. Mycologie concréte: Genera I. Bull Soc. Mycol. France 68: 181-223. van B e ver w i j k A. L. 1951. Zalewski's 'Clathrosphaera spirifera'. Trans. Br. Mycol. Soc. 34: $280-290$.

B 1 ith e n I. 1984. The aquatic stauroconidial Hyphomycetes of Norway with notes on the Nordic species. Nord. J. Bot. 4: $375-392$.

Carmichael J. W., Kendrick W. B, Conaers L. L., S i gler L. 1980. Genera of Hyphomycetes. Alberta Press: Edmonton, 386 pp.

$\mathrm{C}$ a r $\mathrm{r}$ is L. M. 1989. Vacciniuen fungi: Helicoma vaccinii sp. nov. Mycotaxon 36 (1): $29-34$. 
Ciferri R., M ontemartini A. 1957. Sui generi Muchmoria Sacc. e Veronaea n. gen. (Dematiaceac, Didymosporae). Atti Ist. bot. Univ, Lab. Crittogam. Pavia, ser. 5 , 15: $67-72$.

C r a ne J. L, D u m o n t K. P. 1975. Hyphomycetes from the West-Indies and Venezuela. Can. J. Bot. 53 (9): $843-851$.

Cra a e J. L., S chokne cht J. D. 1981. Revision of Torula species. Pseudoaegerita corticalis, Taeniolina deightonii and Xylohypha bowdichiae. Mycologia 73: 78-87.

$\mathrm{Czeczuga}$ B. 1992. Aquatic fungi in the water of melting snow. Acta Mycol. 27: 257-265.

$\mathrm{Czeczuga}$ B. 1995. Hydromycoflora fungi of small forest lakes "Suchary" in the Wigry National Park. Acta Mycol. 30: 167-180.

Czeczuga B, Muszy ti k a E, Pa a kiewicz A. 1999. Zoosporic fungi in the ice of some water reservoirs. Acta Soc. Bot. Pol. 68: 143-147.

Czeczuga B, Orlowska M. 1993. Hyphomycetes in the river Supraśl in various seasons of the year with reference to environmental conditions. Int. Revuc ges. Hydrobiol. 78: $611-630$.

Czeczuga B., Or low \& k a M. 1994. Some aquatic fungi Hyphomycetes on tree leaves. Ann. Acad. Med. Bialostocensis 39: 86-92.

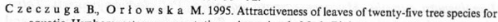
aquatic Hyphomycetes representatives. Ann. Acad. Med. Bialostocensis 40: 233-242.

Czcczuga B. Orłowska M. 1996. Hyphomycetes in twenty springs of the Knyszyn-Bialystok Forest in various seasons. Int. Revue ges Hydrobiol. 81: $417-433$.

$\mathrm{Cz} e \mathrm{czuga}$, $\mathrm{Orlowsk}$ a M. 1997a. Hyphomycetes in morning dew of meadows in northcastern Poland. Ann. Acad. Med. Bialostocensis 42: 187-192.

Czeczuga B., O r łowska M. 1997b. Hyphomycetes in the water of peatmoss (Sphagmom). Ann. Acad. Med. Bialostocensis 42: 193-200.

Czeczuga B., Orlowska M., Wor now icz L. 1989. Some rare species Hyphomycetes in north-eastern Poland. Acta Mycol. 25: 5-20.

Czeczuga B, Ortowska M., Wor on wicz L. 1997. Fungi in water reservoirs of the Western Suwaki Lake District and in some adjacent water basins. Ann. Acad. Med. Bialostocensis $42: 43-63$.

Descals E., N a wawi A., W e b ste r J. 1976. Developmental studies in Actinospora and three similar aquatic hyphomycetes. Trans. Br. Mycol. Soc. 67: 207-220.

D es C a Is E. C, W e b s t e r J.1976. Hyaloscypha: perfect state of Clathrosphaerina zalewskii. Trans. Br. Mycol. Soc. 67: 525-528.

D u dk a I. O. 1974. Wodni hifomiceti Ukraini. Naukova Dumka, Kiev, 239 pp.

D udk a I. A. 1985 . Vodnyje nesovershennyje griby SSSR. Naukova Dumke, Kiev, 187 pp.

E11 is M. B. 1976. More Dematiaceous Hyphomycetes. Commonwelth Mycological Institute, Kew, 507 pp.

Ell is J. B., Ever h a r t B. M. 1891. New species of fungi from various localities. Proc. Acad. Nat. Sci. Philadelphia 43: 76-93.

F a b re E. 1996. Relationships between aquatic hyphomycetes communities and reparian vegetation in 3 Pyrenean streams. Cr. Acad. Sci, Ser. 3: 107-111.

Fis her P. J, P etrin i O., We b s t e r J. 1991. Aquatic hyphomycetes and other fungi in living aquatic and terrestrial roots of Alnus giutinosa. Mycol. Res, 95: 543-547.

Gol terma n H. L., C I y mo R. S. 1969. Methods for chemical analysis of fresh water. Blackwell Scientific Publications, Oxford, $166 \mathrm{pp}$.

G ö n c z 01 J, R é v a y A. 1983. Observations on the hyphomycetes inhabiting forest litter of Hungary. Acta Bot. Hung. 29: 107-125.

Holubova-Jechova V., Mercado, Sierra A. 1984. Studies on Hyphomy. cetes from Cuba II. Hyphomycetes from the Isla de la Juventud. Ceska Mycol. 38: $96-120$.

H ughes S. J. 1951. Studies on micro-fungi. VI. Ceratosporium, Hirudinaria and Hippo. crepidium. Mycol. Pap. 39: 1-24. 
$\mathrm{H}$ u g h es S. J. 1958. Revisiones hyphomycetum aliquot cum appendice de nominibus rejiciendis. Can. J. Bot. 36: 727-836.

I n g o I d C. T. 1942, Aquatic Hyphomycetes of decaying alder leaves. Trans. Br. Mycol. Soc. 25: $339-417$.

I n g o 1 d C. T. 1975. An illustrated guide to aquatic and water born Hyphomycetes (Fungi Imperfecti) with notes on their biology. Fresh. Biolog. Assoc. Sci. Publ. 30: 1-96.

I n gold C. T., C o x V. J. 1957. Heliscus stellatus sp. nov an aquatic hyphomycete. Trans. Br. Mycol. Soc. 40: 155-158.

K i r k P. M. 1984. Volutellaria laurina Tassi, an earlier name for Wiesneriomyces javanicus Koorders. Trans. Br. Mycol. Soc. 82: $748-749$.

$\mathrm{K}$ o ba y a s i $Y_{r}, \mathrm{Fuk}$ u s h $\mathrm{m}$ a H. 1952. On the red and green snow newly found in Japan. Bot Mag. Tokyo 65: 77-85.

Kuthubutheen A. J, $\mathrm{Naw}$ a wi A. 1988. A new species of Wiesneriomyces (Hyphomycetes) from submerged decaying leaves. Trans. Br. Mycol. Soc. 90: 619-625.

K ut hubuthe a A. J., N a wa w i A. 1994. Paracryptophiale kamaruddinil gen. et. sp. nov. from submerged litter in Malaysia. Mycol. Res. 98: 125-126.

$\mathrm{Man}$ o har a C hat y Ch., R a ma r a P. 1972. Stemphyliomma terricola sp. nov, from pond mud. Curr. Sci. 41: 718-719.

M a r v a o vá L. 1973. Notes on Lateriramulosa uni-inflata. Trans. Br. Mycol. Soc. 60 (1): $145-147$.

$\mathrm{M}$ a t s u s h i m a T. 1971. Some interesting Fungi imperfecti. - In: Mycological reports from New Guinea and Solomon Islands (I-II). Bull. Nat. Sei. Mus., Tokyo 14: $460-480$.

Mat s us hi m a T. 1975. Icones microfungorum a Matsushima lectorum. Published by the author: Kobe, 209 pp.

M a t $\mathrm{s}$ u s h i m a T. 1993. Matsushima Mycological Memoirs No 7. Published by the author, Kobe, $75 \mathrm{pp}$.

M or a u F, M o r e a $\mathrm{F}$. 1949. Formes normandes de I'Hellscus lugdunensis Saccardo et Therry. Botaniste 34: $321-327$.

M or g a n-J on es G. 1973. Notes on Hyphomycetes. XVII. A new species of Pseudospirapes. Mycotaxon 5 (2): $481-483$.

N a w a w i A. 1987. Diplocladiella appendiculata sp. nov. a new aero-aquatic hyphomycete. Mycotaxon 18 (2): 297-302.

Nawawi A, K a th u buthe n A. J. 1988. Beverwykella cerebriformis sp. nov. an aero-aquatic hyphomycete from Malaysia. Trans. Br. Mycol. Soc. 90: 487-491.

$\mathrm{N}$ il s s o n S. 1964. Freshwater Hyphomycetes. Taxonomy, morphology and ecology. Symb. Bot. Uppsal. 18: 1-130.

R u t t n o F. 1962. Grundriss der Limnologie. Aufl. 3. Walter de Gruyter, Berlin, 332 pp. $\mathrm{S}$ a c c a rd o P. A. 1880. Conspectus generum fungorum Italiae inferiorum nampe ad Sphaeropsdeas, Melanconieas et Hyphomyceteas pertinentium systemate sporologico dispositorum. Michelia 2: $1-39$.

Sa nders P. F, Webster J. 1978. Survival of aquatic hyphomycetes in terrestrial situations. Trans. Br. Mycol. Soc. 71: 231-237.

S p e g a z i a C. 1918. Notes micológicus. Physis (Buenos Aires) 4: 281-295.

$\mathrm{S} \mathrm{hridhar} \mathrm{K.} \mathrm{R,} \mathrm{Ka} \mathrm{veria} \mathrm{p} \mathrm{p} \mathrm{a} \mathrm{K.} \mathrm{M.} \mathrm{1988.} \mathrm{Survival} \mathrm{of} \mathrm{water-borne} \mathrm{fungi} \mathrm{imperfecti}$ under non-aquatic conditions. Proc. Indian Nat. Sci. Acad. B 54: 295-297.

S u b r a m a n i a C. V., L o d h a B.C. 1964. Four new coprophilous Hyphomycetes. Ant. v. Leeuwenhoek $30: 317-330$.

Sutto n B. C. 1984. Notes on Titaea (Hyphomycetes). Trans. Br. Mycol. Soc. 83: $399-413$.

S y d ow H. 1902. Novae fungorum species. Ann. Mycol. 1: 54-65.

T ót h S. 1973. Vizi hyphomycetes adatok. Erdèlyböl. Bot. Kōzl. 60: 13-18.

T u ba k i K. 1960 . On the Japanese Hyphomycetes. Scum and foam group, referring to the preliminary survey of the snow group. Nagaoa 7: 15-29. 
T u ba k i K., T oku ma s u S, A n d o K. 1985. Morning dew and Tripospermum (Hyphomycetes). Bot. J. Linnean Soc. 91: 45-50.

T ze a n S. S., C h e n J. L. 1991. Two new species of Dactylaria from Taiwan. Mycol. Res. 95: $1000-1004$.

Z a l e w s k i A. 1888. Przyczynki do życioznawstwa grzybów. L Clathrosphaera spirifera. Rozpr. i Sprawozd. z Posiedzeri Wydziału Mat.Przyrod. Kraków 18: 153-190.

Hyphomycetes w wodzie deszczowcj oraz w wodzie z topniejącego śniegu i lodu

Streszezenie

Przeprowadzone badania w warunkach laboratoryjnych metodą bezpośredniego mikroskopowania oraz metodą przynẹt (resztki roślinne, celofan i wylinka węża) w wodzie deszczowej spływającej z drzew, z dachów o różnym pokryciu oraz w stopniałym śniegu i lodzic pozwoliły ustalić obecność 146 gatunków grzybów klasy Hyphomycetes, w tym 55 nowych dla Polski.

Wśód oznaczonych grzybów dominowały tak zwane gatunki powictrzno-wodne, a tylko kilkanaście należało do typowo wodnych. 